\title{
ANALYSIS OF THE LOADING ANIMATION PERFORMANCE AND VIEWER PERCEPTION
}

\author{
Gojko Vladić (D), Selena Mijatović, Gordana Bošnjaković (D), \\ Ivana Jurič (D), Vladimir Dimovski (iD) \\ University of Novi Sad, Faculty of Technical Sciences, \\ Department of Graphic Engineering and Design, Novi Sad, Serbia
}

\begin{abstract}
Digital content presented to the viewer usually has to be processed by the device on which is displayed, in case of internet content processing is done by hosting server and user device with additional download time. Time elapsed for these tasks differs depending on the quantity of the data and complexity of the processing needed. Waiting time for content to be displayed can have significant influence on the user experience. Loading animations are often used to divert viewers' attention or to provide viewer with the information about the process progress, estimated time, etc. Performance of these animation can differ depending on their type, elements or even a story. This paper presents analysis of the performance and viewer perception of different loading animations. Survey and eye tracking were used to gain insight in to the viewer's perception of the loading animation. Results show noticeable differences caused by loading animation type.
\end{abstract}

Key words: Loading animation, User experience, Perceived Performance

\section{INTRODUCTION}

Many of everyday human tasks are infused with digital content. It can be argued that whole humanity today, with some exemptions, is highly accustomed to digital content consumption. Digital content has to be processed by the user device. If the content is distributed through the internet the content is processed by the provider servers and users device. All of these processing tasks take time, additionally internet transfer of the data takes time too. Sum of time that all of these tasks take is called loading time. User experience can be influenced by the digital content loading time. User experience (UX) is how the user interacts with a product, and the emotion and experiences this interaction creates (Persson, 2019). Google published a study in 2017 establishing relation between loading time and bounce rate by the web site viewers.

Results of this study reveal that (Google, 2020):

- $1-3$ seconds load time increase the bounce rate probability by $32 \%$

- $1-5$ seconds load time increase the bounce rate probability by $90 \%$

- 1-6 seconds load time increase the bounce rate probability by $106 \%$

- $1-10$ seconds load time increase the bounce rate probability by $123 \%$

This Google's study confirmed facts about human perceptual abilities, first established by Miller 1968, and others later (Card et al, 1991; Miller, 1968; Nielsen, 1993).

There are 3 main human perceptual time limits:

- 0.1 second makes users feel instantaneous,

- $\quad 0.1-1$ second makes users realize the delay and feel of non-operating directly,

- $\quad 1-10$ seconds need to provide feedback for users to reduce impatience.

There is a significant difference between loading time and perceived loading time. It is well known that people perceive time differently while waiting compared to when performing an action. Action can be anything that occupies viewers' attention. One of the most used tools for occupying viewers' attention is loading animation, although a simple game or some other activity can be used also.

Animation today is not limited to only animated series and movies. It is often used in applications, websites and other various digital services and tools in order to improve the appeal, usability, or to indicate a change. However, there is currently a lack of well-regarded guidelines for animation in relation to user interfaces (UI) on the different digital platforms (i.e. any type of computers, including smartphones and tablets) (Persson, 2019). 
Loading animation can inform users about the expected wait time, explanation of the process, reason for waiting, indicate status, such as downloading, uploading or saving data. There are several approaches to the loading animation design. Progress indicators inform the user that an operation is in progress. They can be classified as determinate and indeterminate progress indicators, depending on whether they present how long a process will take, or just inform the viewer that process is in progress with uncertain amount of wait time. Graphic presentation of the progress indicator is limited only by the creativity of the designer and the technology used for loading animation production (Hao, 2020). Usual and well known approaches to loading animation design is circular or linear progress indicator, Figure 1.
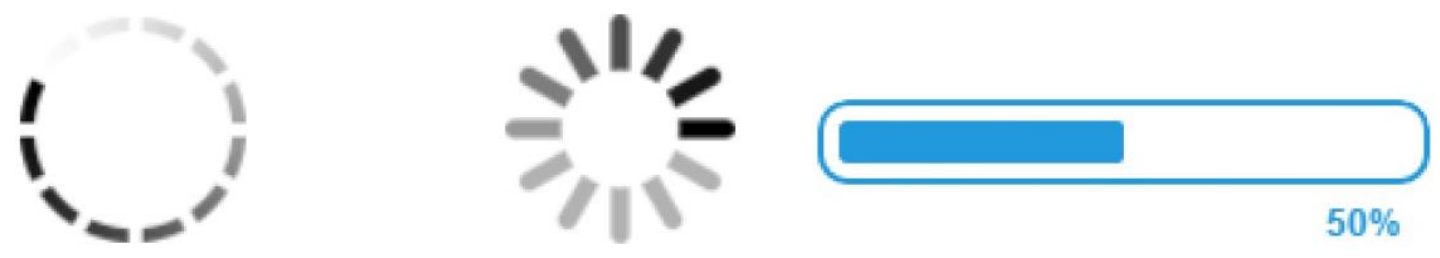

Figure 1: Circular and linear progress indicator

On the other hand they are not too useful for occupying viewer attention for a long time, as they are repetitive and simple. Loading animation with the "story", shown in figure 2, are far more engaging for the viewer, resulting in different perception of the time elapsed.
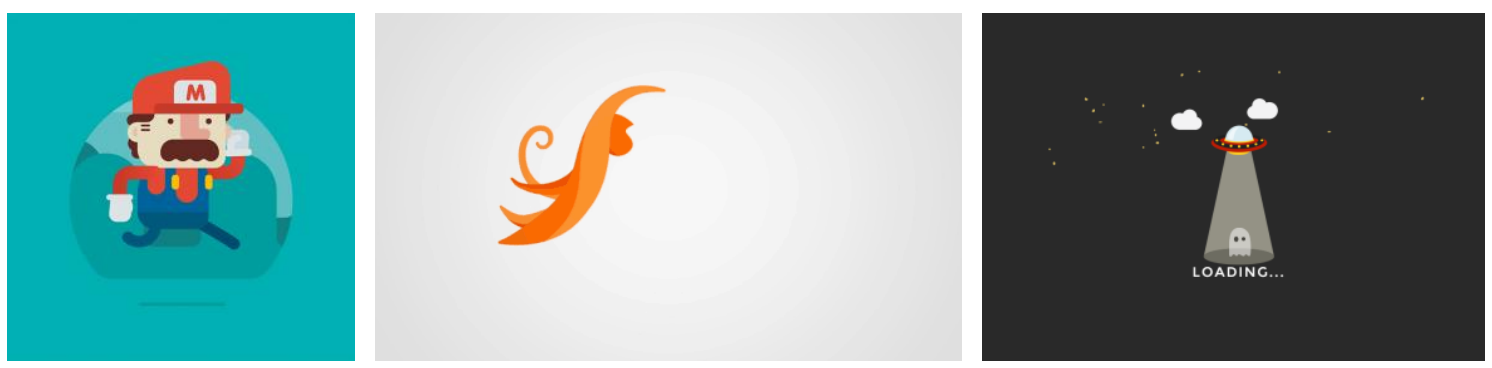

Figure 2: Loading animations with the "story"

This paper aims to examine influence of the loading animation complexity, used elements and variations of those elements on the viewer's perception of the loading animation.

\section{METHODS}

In order to determine influence of loading animation content and variations of its elements, 17 different animations were prepared using Adobe After Effects and Cinema 4D. There were 2D animation (figure 3), faux 3D animations (2D animation that look like 3D, figures 4-8) and real 3D animation (figure 9). Animations had variations concerning shape of the elements, color, content (text, loading bar), and complexity. All animations were shown in the same duration, 10 seconds, where some of them repeated several times depending on the cycle length (loop animation), while other run only once,

Survey was conducted in order to gather insight in to viewer's perception of selected animations. Animations were shown in randomized order with accompanying question about subjective perception of the duration. Aside the survey, eye tracking experiment was done in order to observe participants viewing patterns of different animations. Gaze Point GP3 device and 24" computer monitor were used for this part of the research.

138 participants submitted their opinion on the shown animations trough the survey, while 10 new participants were included in the eye tracking part of the experiment. $64 \%$ were female and $36 \%$ male participants. Majority of them were 25 to 50 years old.

All participants were instructed to give their unbiased opinion, without using any strategies in their answers. 


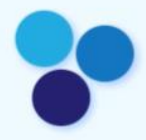

a)

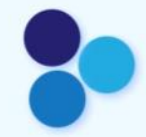

LOADING...

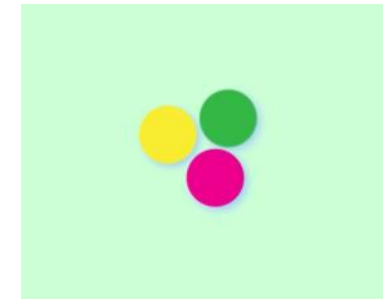

c)

Figure 3: 2D animation: a) circles in circular motion, b) added text, c) color variation

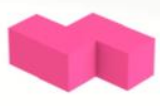

a)

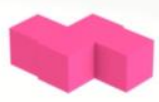

RQDDIEGoes

b)

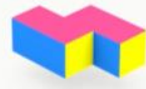

c)

Figure 4: Faux 3D animation: a) rotating bricks, b) added text, c) color variation

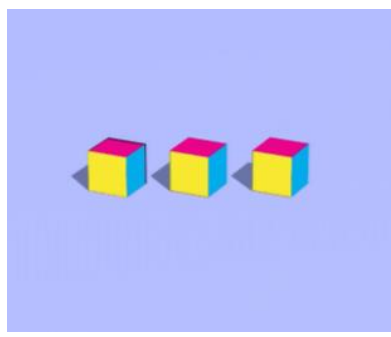

a)

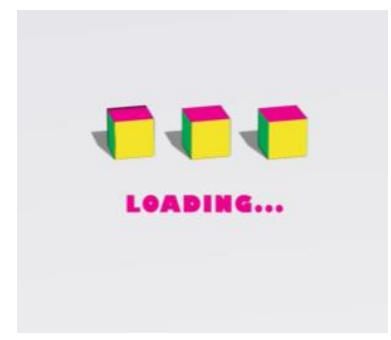

b)

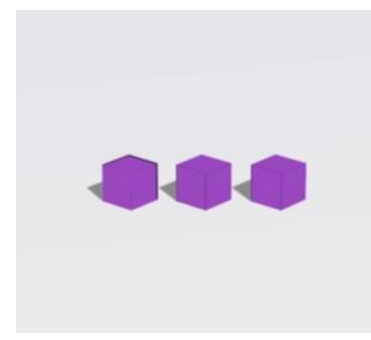

c)

Figure 5: Faux 3D animation: a) circulating boxes, b) added text, c) color variation

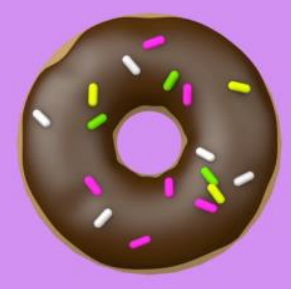

a)

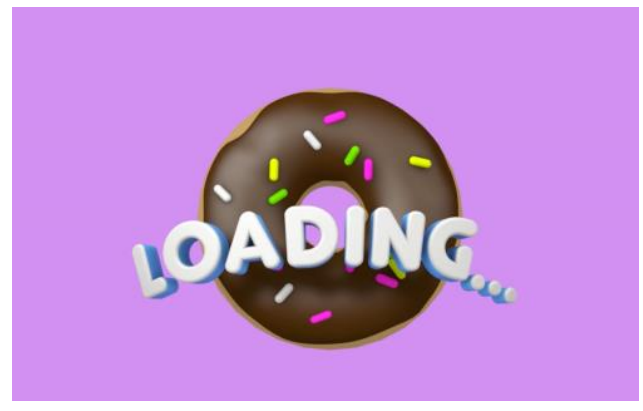

c)

Figure 6: 3D animation appearing donut: a) without text, b) with text 


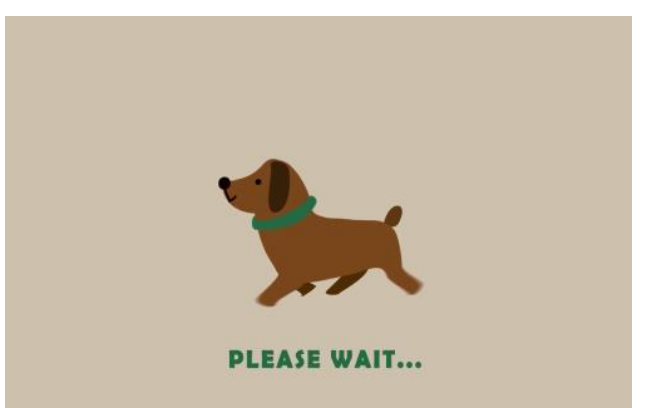

a)

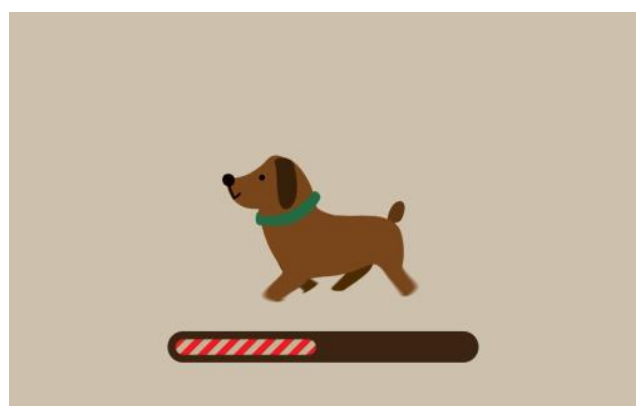

c)

Figure 7: 2D animation of walking dog: a) with text, b) with progress bar

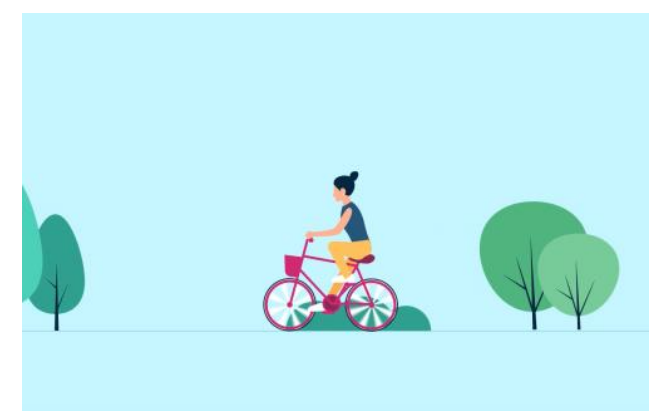

a)

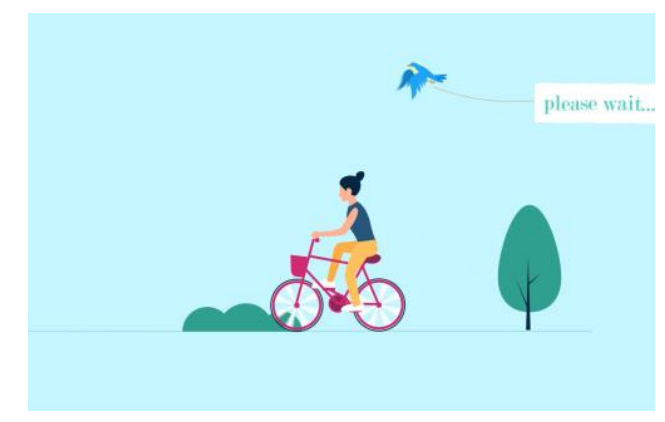

c)

Figure 8: 2D animation of bicycle ride: a) without text, b) with text

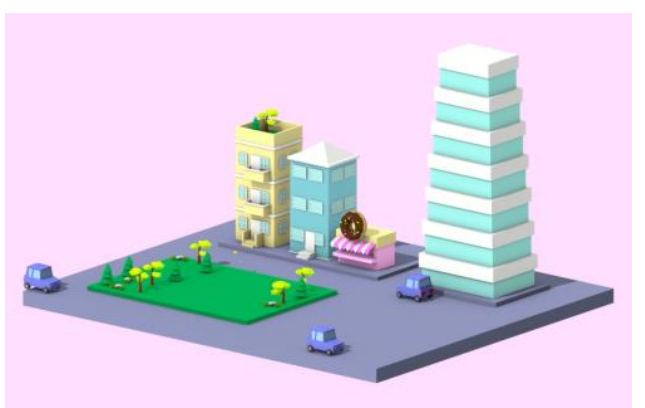

a)

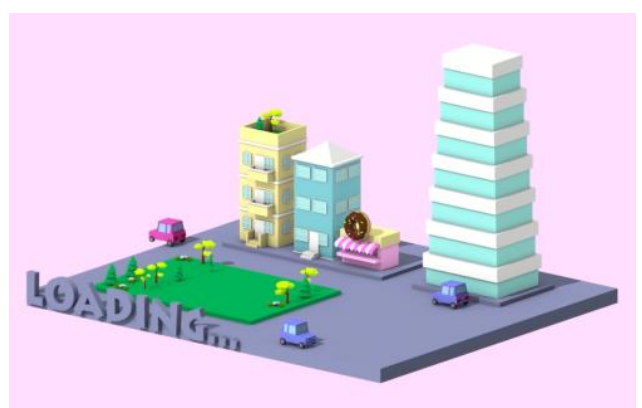

c)

Figure 9: 3D animation of appearing city: a) without text, b) with text

\section{RESULTS AND DISCUSSION}

\subsection{Perceived duration of the animation / loading speed}

Results of the survey revealed that simple loop animations were judged as not slow or fast loading (duration of animation / speed of loading) by majority of the participants. Figure 10 shows results for the simple 2D animation with circles in circular motion. All the simple geometrical animations showed similar pattern with small deviations. Differences in the animation elements (text and color) did not influence perceived duration of the animation. 


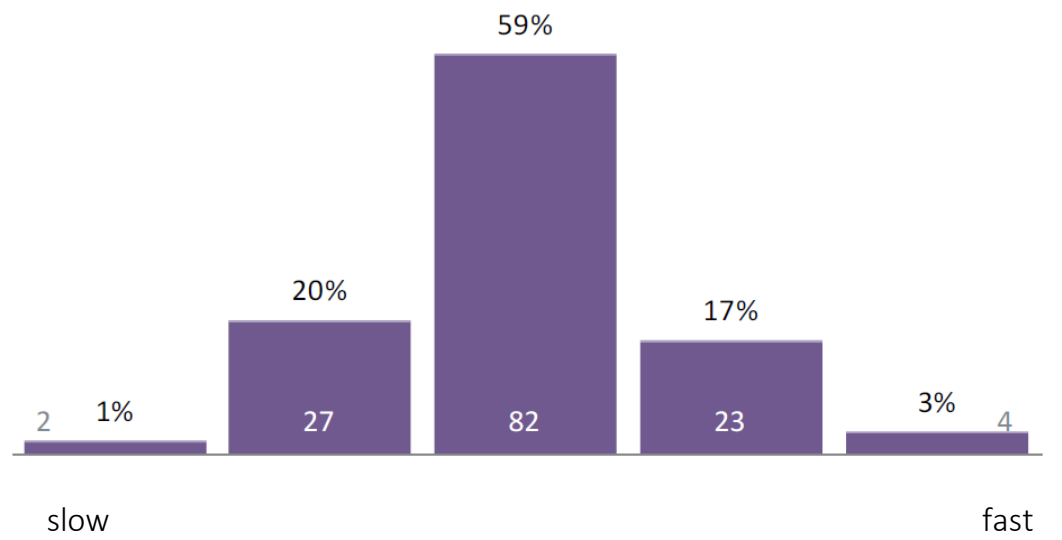

Figure 10: Results for the simple 2D animation with circles in circular motion duration of animation /speed of loading

Variations in the 2D animation of walking dog with text and with progress bar showed a difference in the perceived duration. Animation with text was perceived as longer. This could be explained by the fact that progress bar offered viewers additional information which occupied their attention and as a result the animation with progress bar was perceived as shorter. Figure 11 presents the differences in perceived duration.

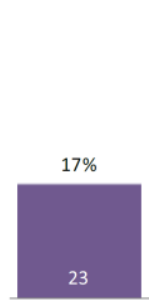

slow

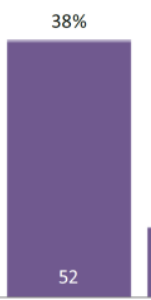

a)

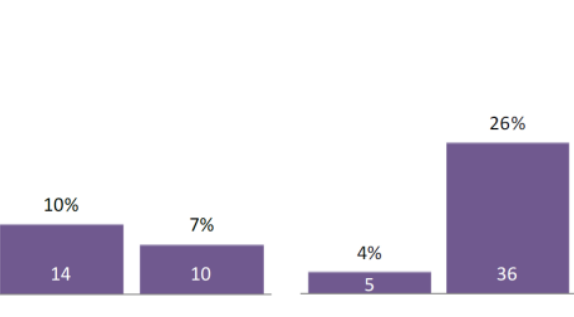

fast slow
$46 \%$
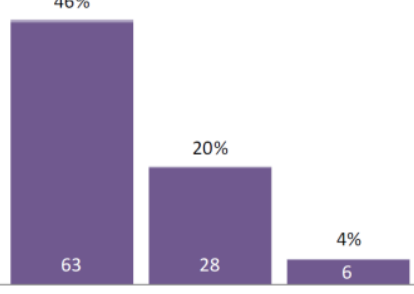

fast

b)

Figure 11: Results for the duration of animation/speed of loading 2D animation of walking dog:

a) with text, b) with progress bar

Similarly, but to lesser extent, as addition of progress bar in the previous case, addition of the text to the 3D animation appearing donut offered more content to the viewer. This in turn resulted in shorter perceived time of the animation with additional text message. Animation of bicycle ride with and without text showed the similar pattern. To be specific, the animation with text was perceived as shorter in duration. Perceived duration of the animation without and with text is shown in Figure 12 for animation appearing donut and Figure 13 for animation bicycle ride.

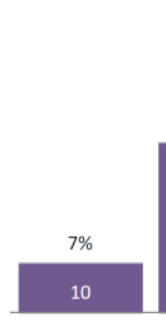

slow
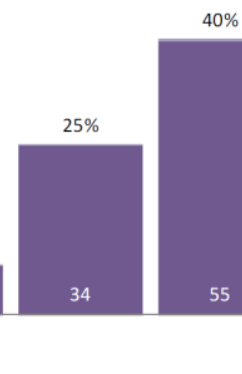

a)

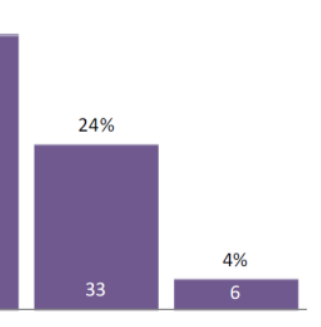

fast

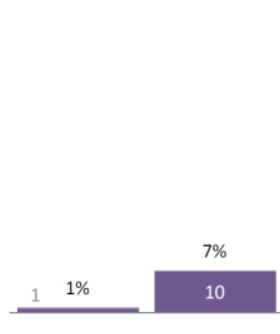

slow

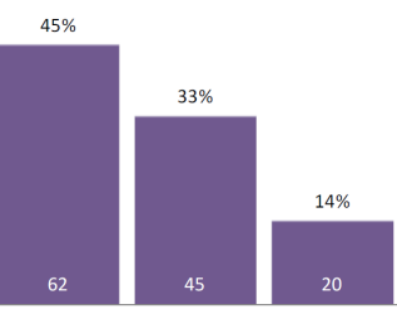

fast

b)

Figure 12: Results for the duration of animation /speed of loading 3D animation of appearing donut: a) without text, b) with text 


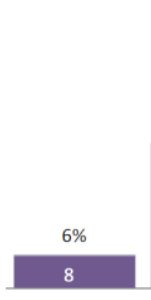

slow

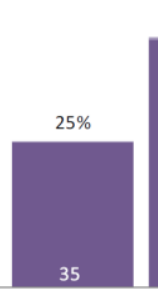

60

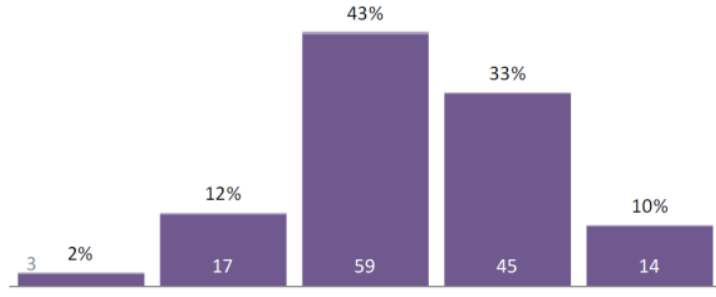

slow

b)

Figure 13: Results for the duration of animation/speed of loading 2D animation of bicycle ride: a) without text, b) with text

Presence of the text in the 3D animation of appearing city did not produce any differences in the perceived duration of the animation. Possibly due to the overall complexity of the animation.

\subsection{Eye tracking results}

Eye tracking recording offered insight in to viewing pattern of the participants. Figures 14-20 show heat map visualization of the viewing pattern of one participant, which was selected as a typical representative. Each participant has unique viewing pattern and in this case summary heat map did not offer good presentation. Figures 13-16 show results for simple animations with variations in the elements. Even though there was no reported difference in the perceived duration, differences in the viewing pattern are obviously present and influenced by the animation elements. In all three simple geometrical animations gaze fixations are much more concentrated in the case of animations with text and scattered in case of animations with just objects moving. Variation of color did not have any influence. In case of these simple animations order in which they are shown to the viewer also had influence. As animations are simple, viewer had more concentrated gaze fixation first time he/she saw the animation. The next time similar animation was shown to the participant gaze fixations were more scattered as if viewer was searching the screen for some information.

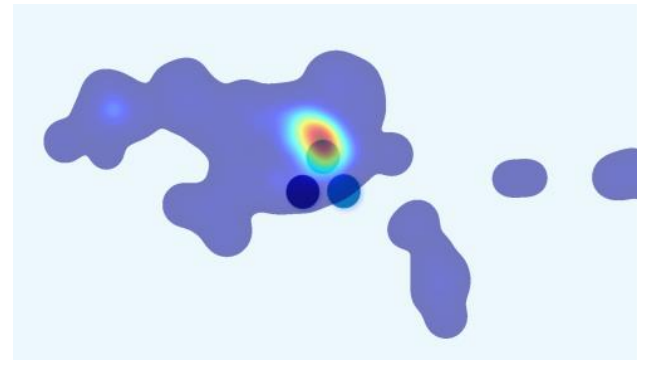

a)

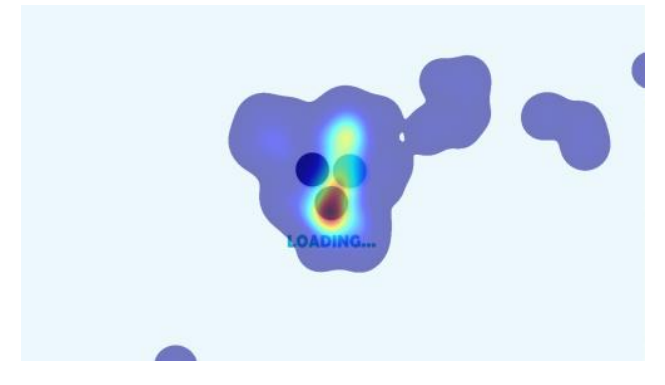

b)

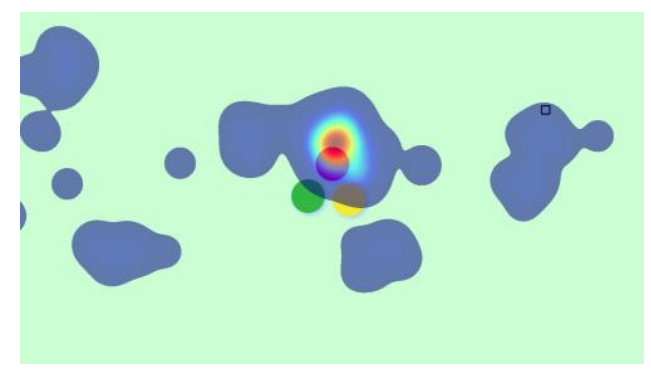

c)

Figure 14: Eye tracking heat map for 2D animation: a) circles in circular motion, b) added text, c) color variation 


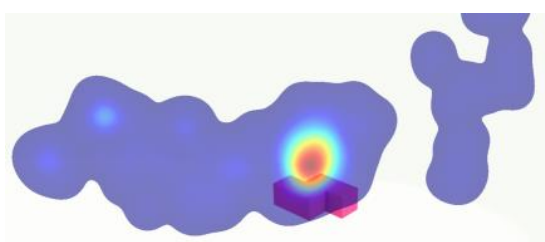

a)

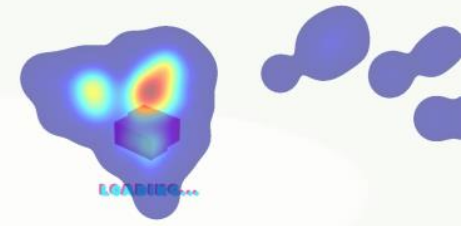

b)

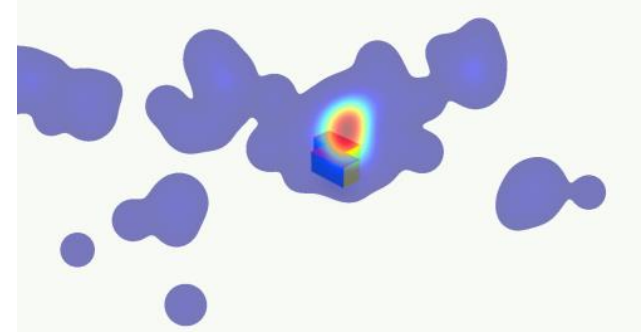

d)

Figure 15: Eye tracking heat map for Faux 3D animation: a) rotating bricks, b) added text, c) color variation

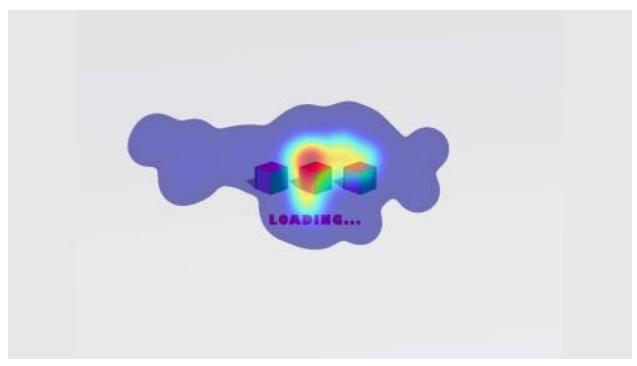

a)

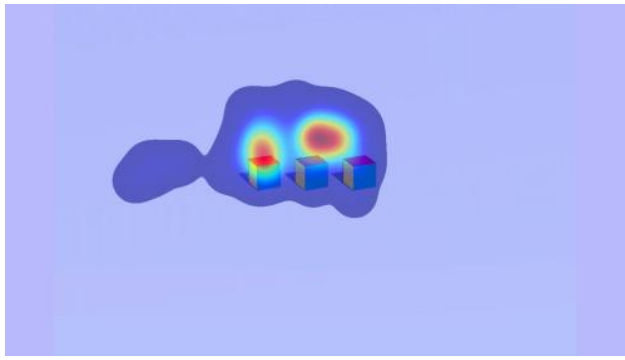

b)

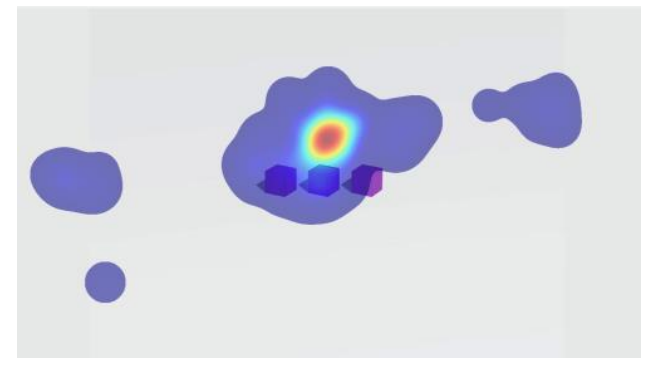

c)

Figure 16: Eye tracking heat map for Faux 3D animation: a) circulating boxes, b) added text, c) colorvariation

Similarly as in the case of simple animations, Figure 17 shows that presence of text concentrates gaze fixations on the animation and viewers are not searching throughout the screen for more information.

Figure 18 shows that presence of the progress bar has a similar effect as the text, as gaze fixations are concentrated. Looking at the heat map Figure 18a, could lead to the conclusion that effect is stronger with progress bar than with text, but results of other participants do not support that clearly. More specialized experiment would be advised.

Eye tracking the viewing of the bicycle ride and appearing city animations show that participants were observing all of the elements of the animations. Whether those elements are distributed all over the screen of concentrated in one area did not make any difference. More complex animations, as these two examples, are packed with elements for the viewer to analyze thru whole duration of the animation. 


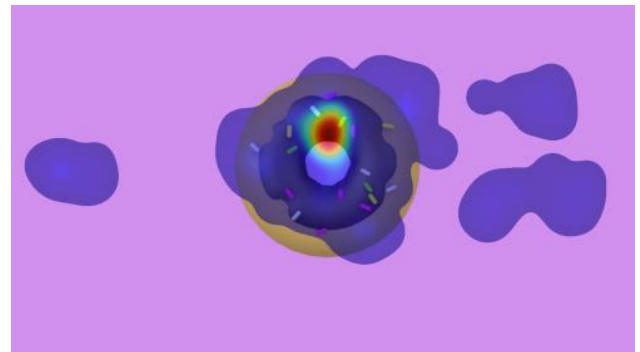

a)

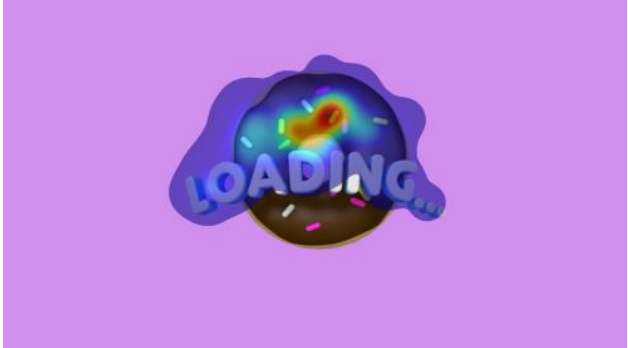

b)

Figure 17: Eye tracking heat map for 3D animation: a) appearing donut, b) added text

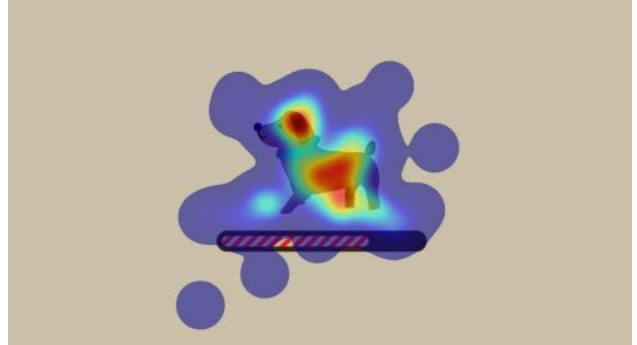

a)

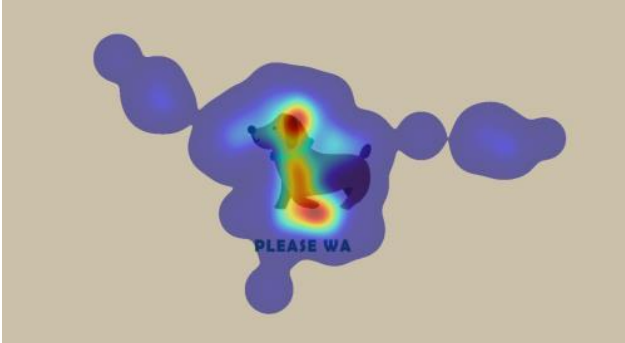

b)

Figure 18: Eye tracking heat map for 2D animation of walking dog: a) with text, b) with progress bar

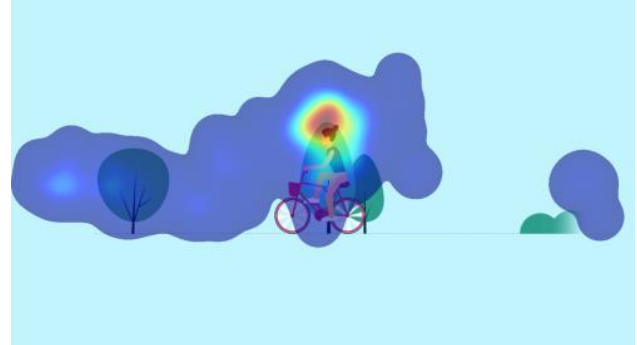

a)

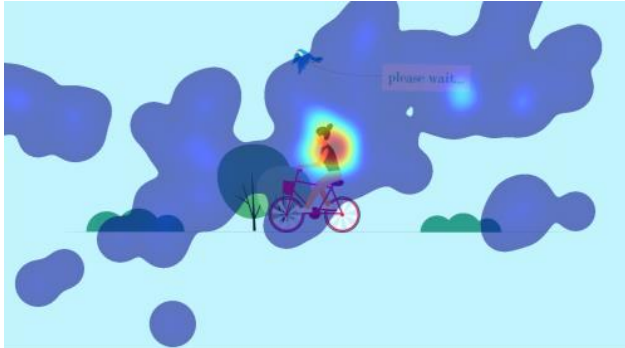

b)

Figure 19: Eye tracking heat map for 2D animation of bicycle ride: a) without text, b) with text

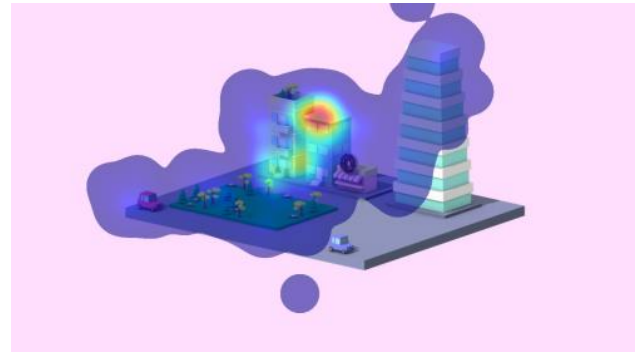

a)

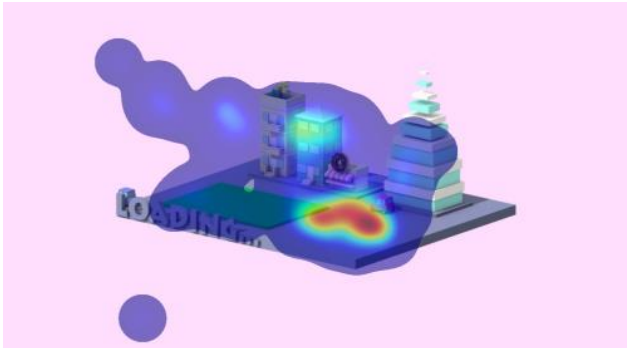

b)

Figure 20: 3D animation of appearing city: a) without text, b) with text 


\section{CONCLUSIONS}

Loading animations play important role in UX design, and must be taken in to consideration if users are waiting longer than 1 second while using the mobile or computer application, web site, etc. Even if waiting is unavoidable it should be made as pleasant as possible for the user. Results of the survey showed that perception of animation duration could be manipulated. Displaying text or progress bar could be used as assets in achieving perception of shorter wait time. The Eye tracing part of the experiment revealed that viewer will search the screen for additional information if animation is offering the same content in a loop. This could be countered by using text which holds the attention of the user for the longer time. Using text or progress bar showed effectiveness in both simple and complex animations. Differences between effects of using progress bar and text were not conclusive and should be examined in more details in future experiments.

\section{ACKNOWLEDGMENTS}

This research (paper) has been supported by the Ministry of Education, Science and Technological Development through the project no. 451-03-68/2020-14/200156: "Innovative scientific and artistic research from the FTS (activity) domain".

\section{REFERENCES}

[1] Card, S. K., Robertson, G. G., Mackinlay, J. D.: "The information visualizer: An information workspace", Proceedings of ACM CHI'91 Conference 1991, (ACM CHI'91: New Orleans, LA, 1991), pages 181-188. doi: 10.1145/108844.108874.

[2] Google API: "Find Out How You Stack Up to New Industry Benchmarks for Mobile Page Speed", URL: https://think.storage.googleapis.com/docs/mobile-page-speed-new-industry-benchmarks.pdf (last request: 2020-09-15).

[3] Hao, Y.: "Understanding loading animations - types and applications", URL: https://uxdesign.cc/understanding-loading-animation-types-and-its-application-e41ba914b847 (last request: 2020-10-14).

[4] Miller, R.: "Response time in man-computer conversational transactions", Proceedings of AFIPS Fall Joint Computer Conference 1968, (AFIPS/ACM/Thomson Book Company, Washington D.C., 1968), pages 267-277, URL:

http://yusufarslan.net/sites/yusufarslan.net/files/upload/content/Miller1968.pdf (last request: 2020-10-14).

[5] Nielsen, J.: "Response times: the three important limits, Excerpt from Chapter 5 of Usability Engineering by Jakob Nielsen", Academic Press, 1993., URL: http://www.useit.com/papers/responsetime.html (last request: 2020-09-15).

[6] Persson, S.: "Improving perceived performance of loading screens through animation", Bachelor thesis, Linnaeus University, Sweden, 2019. URL: https://www.divaportal.org/smash/get/diva2:1333185/FULLTEXT01.pdf (last request: 2020-10-14).

[7] Preece, J., Rogers, Y., Sharp, H.: "Interaction Design: Beyond human-computer interaction", 4th edition, (Wiley: Chichester, 2015.).

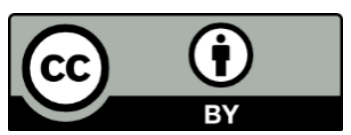

(C) 2020 Authors. Published by the University of Novi Sad, Faculty of Technical Sciences, Department of Graphic Engineering and Design. This article is an open access article distributed under the terms and conditions of the Creative Commons Attribution license 3.0 Serbia (http://creativecommons.org/licenses/by/3.0/rs/). 